\title{
On the importance of the wind emission to the optical continuum of OB supergiants
}

\author{
M. Kraus ${ }^{1}$, J. Kubát ${ }^{1}$, and J. Krtička ${ }^{2}$ \\ 1 Astronomický ústav, Akademie věd České republiky, Fričova 298, 25165 Ondřejov, Czech Republic \\ e-mail: kraus@sunstel.asu.cas.cz; kubat@sunstel.asu.cas.cz \\ 2 Ústav teoretické fyziky a astrofyziky PřF MU, 61137 Brno, Czech Republic \\ e-mail: krticka@physics.muni.cz
}

Received 5 November 2007 / Accepted 15 January 2008

\begin{abstract}
Context. Thermal wind emission in the form of free-free and free-bound emission is known to show up in the infrared and radio continuum of hot and massive stars. For OB supergiants with moderate mass loss rates and a wind velocity distribution with $\beta \simeq 0.8 \ldots 1.0$, no influence of the wind to the optical continuum, i.e. for $\lambda \lesssim 1.0 \mu \mathrm{m}$, is expected. Investigations of stellar and wind parameters of OB supergiants over the last few years suggest, however, that for many objects $\beta$ is much higher than 1.0, reaching values up to 3.5. Aims. We investigate the influence of the free-free and free-bound emission on the emerging radiation, especially at optical wavelengths, from $\mathrm{OB}$ supergiants having wind velocity distributions with $\beta \geq 1.0$.

Methods. For the case of a spherically symmetric, isothermal wind in local thermodynamical equilibrium (LTE) we calculate the freefree and free-bound processes and the emerging wind and total continuum spectra. We localize the generation region of the optical wind continuum and especially focus on the influence of a $\beta$-type wind velocity distribution with $\beta>1$ on the formation of the wind continuum at optical wavelengths.

Results. The optical wind continuum is found to be generated within about $2 R_{*}$ which is exactly the wind region where $\beta$ strongly influences the density distribution. We find that for $\beta>1$, the continuum of a typical OB supergiant can indeed be contaminated with thermal wind emission, even at optical wavelengths. The strong increase in the optical wind emission is dominantly produced by free-bound processes.
\end{abstract}

Key words. stars: early-type - stars: supergiants - stars: winds, outflows - stars: mass-loss - stars: circumstellar matter

\section{Introduction}

It is well established that for massive stars the appearance of (thermal) excess emission at infrared (IR) and radio wavelengths is caused by free-free and (to a small fraction also by) free-bound emission generated in their winds (see e.g. Panagia \& Felli 1975; Olnon 1975). At radio wavelengths, the free-free excess emission is usually used to derive the mass loss rates of hot and massive stars (see e.g. Lamers \& Leitherer 1993; Puls et al. 1996).

Waters \& Lamers (1984) have investigated this excess emission in detail, especially in the near-IR region. These authors studied the influence of free-free and free-bound emission to the total continuum and pointed already to the importance of the wind velocity (and hence the wind density) distribution that can severely alter the wind contribution in the IR.

However, the investigations of Waters \& Lamers (1984) were restricted to the IR and radio range, i.e. they calculated the wind contribution for $\lambda \gtrsim 1 \mu \mathrm{m}$ only, while during earlier studies Brussaard \& van de Hulst (1962) had noted that free-bound processes might become very important in the optical and UV range for temperatures typically found in the winds of hot stars and supergiants.

Whether the free-bound emission in the wind indeed influences the optical continuum, depends severely on the density distribution. For line-driven winds, the density follows from the equation of mass continuity, i.e. it is proportional to the mass loss rate, and inversely proportional to the wind velocity. A high wind density can therefore be reached by either a high mass loss rate, or a low wind velocity.

For massive stars with pronounced high mass loss rates like Wolf-Rayet stars, Luminous Blue Variables, or the group of $\mathrm{B}[\mathrm{e}]$ stars, it is well known that the wind not only influences, but even dominates, the optical spectrum. In these objects, the wind is usually optically thick even in the visual range and thus completely hides the stellar spectrum. Some recent examples of the thermal wind influence in the form of free-free and free-bound emission at optical wavelengths have been published, e.g. by Guo \& Li (2007) for the case of Luminous Blue Variables, and by Kraus et al. (2007) for a Magellanic Cloud B[e] supergiant.

The second density triggering parameter is the wind velocity distribution. For OB-type stars with line-driven winds, the wind velocity is very often approximated by a so-called $\beta$-law, where $\beta$ is in the range of $0.8 \ldots 1.0$. Such a $\beta$ value causes a rather fast wind acceleration at the base of the wind, and the wind reaches its terminal value within a few stellar radii (see e.g. Lamers \& Cassinelli 1999). Consequently, the region of very high density that might cause enhanced free-bound emission is restricted to an extremely small volume around the stellar surface. We can, therefore, expect that for OB-type stars, which have only moderate mass loss rates and whose wind velocity distributions have $\beta$ values between 0.8 and 1.0, there will be no noticable influence of the wind on their optical continuum emission.

During the last few years, huge effort has been made to determine precisely the stellar and wind parameters of OB-type 
supergiants in the Galaxy (e.g. Kudritzki et al. 1999; Markova et al. 2004; Fullerton et al. 2006; Crowther et al. 2006; Puls et al. 2006), in the Magellanic Clouds (see e.g. Evans et al. 2004; Trundle et al. 2004; Trundle \& Lennon 2005), and beyond, e.g., in M 31 (e.g. Bresolin et al. 2002). Interestingly, many OB supergiants are found to have rather high $\beta$ values (up to $\beta=3.5$, see Sect. 3.2). Such high $\beta$-values, resulting in a strong density increase close to the stellar surface due to a much slower wind acceleration, should have noticable effects on the thermal wind emission not even in the near-IR, but extending also to optical wavelengths. In this paper, we, therefore, aim to investigate and discuss in detail the influence of high $\beta$ values on the wind emission of OB supergiants, especially at optical wavelengths.

\section{Description of the model OB supergiant}

The calculation of the continuum emission of a typical OB supergiant is performed in three steps: (i) first we calculate the stellar emission of the supergiant with no stellar wind; (ii) then, we calculate the emission of the wind with the stellar parameters as boundary conditions; (iii) and finally, we combine the two continuum sources whereby the stellar emission still has to pass through the absorbing wind. A justification of the use of this so-called core-halo approximation together with a discussion of several other assumptions and simplifications are given in Sect. 4.

\subsection{The stellar model}

To simulate a typical OB supergiant we adopt the following set of stellar parameters: $T_{\text {eff }}=33000 \mathrm{~K} ; R_{*}=17.2 R_{\odot}$; $\log L_{*} / L_{\odot}=5.5$; and $\log g=3.4$. With these parameters, we compute the stellar continuum emission of a hydrogen plus helium atmosphere, given by the Eddington flux, $H_{v}$. These calculations are performed with the code of Kubát (2003, and references therein), which is suitable for the calculation of non-LTE spherically-symmetric model atmospheres in hydrostatic and radiative equilibrium.

\subsection{The wind model}

For simplification, we assume that hydrogen in the wind is fully ionized while further contributions to the electron density distribution from, e.g., helium and the metals, are neglected. This means that for a given mass loss rate the real number density of free electrons is underestimated. This leads to an underestimation of the total wind emission generated via free-free and freebound processes. However, since we are interested only in the effect on the emerging wind emission caused by different velocity distributions, such a simplification is reasonable. We further neglect electron scattering, and describe the wind zone following Panagia \& Felli (1975) with a spherically symmetric stationary model in LTE. We show the frame of reference used for our computations in Fig. 1. The electron number density distribution, $n_{\mathrm{e}}(r)$, then equals the hydrogen number density distribution, $n_{\mathrm{H}}(r)$, which follows from the equation of mass continuity,

$n_{\mathrm{e}}(r)=n_{\mathrm{H}}(r)=\frac{\dot{M}}{4 \pi \mu m_{\mathrm{H}} r^{2} v(r)}$.

This equation relates the density at any location $r$ in the wind to the mass loss rate, $\dot{M}$, of the star and the wind velocity, $v(r)$. The parameters $\mu$ and $m_{\mathrm{H}}$ are the mean atomic weight, for which

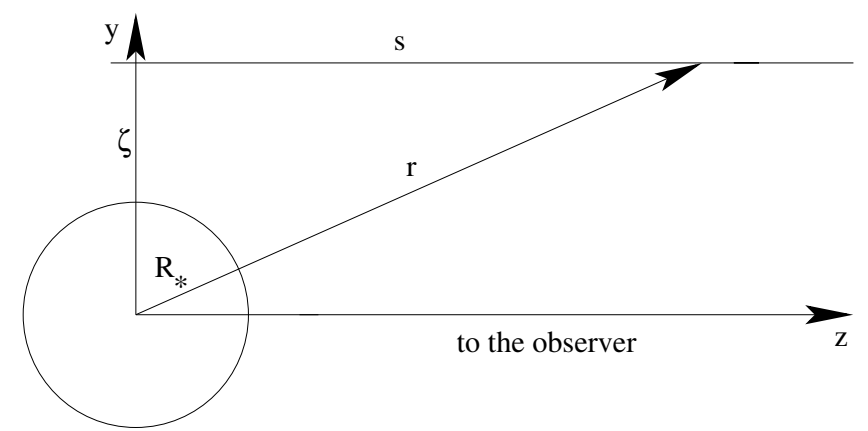

Fig. 1. Frame of reference used for the calculations (after Panagia \& Felli 1975). The $x$-axis is perpendicular to the drawing-plane.

we use a value of 1.4 (i.e. solar composition), and the atomic hydrogen mass, respectively.

The velocity increase in line-driven winds of hot stars is approximated with a $\beta$-law of the form (see e.g. Lamers \& Cassinelli 1999)

$v(r)=v_{0}+\left(v_{\infty}-v_{0}\right)\left(1-\frac{R_{*}}{r}\right)^{\beta}$

where $R_{*}$ is the stellar radius, $v_{\infty}$ is wind terminal velocity, and $\beta$ describes the "steepness" of the velocity increase at the base of the wind. The term $v_{0}$ gives the velocity at the base of the wind, i.e., at $r=R_{*}$, for which we use the thermal hydrogen velocity given by the wind temperature.

The wind temperature for hot stars is known to drop quickly with distance (see e.g., Drew 1989), and a useful description of the radial electron temperature distribution as a function of effective temperature and wind velocity has been derived by Bunn \& Drew (1992)

$T_{\mathrm{e}}(r)=0.79 T_{\mathrm{eff}}-0.51 \frac{v(r)}{v_{\infty}} T_{\mathrm{eff}}$.

This relation states that in the vicinity of the stellar surface, the electron temperature is roughly $0.8 T_{\text {eff }}$, a value that has been found and confirmed by other investigators as well (see e.g. Drew 1989; de Koter 1993; Krtička 2006).

For the purpose of our investigation, it is sufficient to restrict the model computations to an isothermal wind, and throughout this paper we will use the value of $T_{\mathrm{e}}=T_{\mathrm{e}}\left(R_{*}\right)=25760 \mathrm{~K}$ (which is lower than $0.8 T_{\text {eff }}$ ) following from Eq. (3) for our star with $T_{\text {eff }}=33000 \mathrm{~K}$. A justification for these assumptions and a discussion of their reliability are given in Sect. 4.1.

The assumption of a constant wind temperature allows for a simplified treatment of the radiation transfer and therefore of the intensity calculation. Consequently, at each impact parameter, $\zeta$ (see Fig. 1), the intensity of the wind emission is given by

$I_{v}=B_{v}\left(T_{\mathrm{e}}\right)\left(1-\mathrm{e}^{-\tau_{v}(\zeta)}\right)$

The optical depth is defined as the line-of-sight integral over the absorption coefficient of the free-free and free-bound processes, $\kappa_{v}(\zeta, s)$

$\tau_{v}(\zeta)=\int_{s_{\min }}^{s_{\max }} \kappa_{v}(\zeta, s) \mathrm{d} s$

with the integration limits

$\begin{array}{llrl}s_{\min }=\sqrt{R_{*}^{2}-\zeta^{2}} & \text { for } & 0 & \leq \zeta<R_{*}, \\ s_{\min }=-\sqrt{R_{\text {out }}^{2}-\zeta^{2}} & \text { for } & R_{*} \leq \zeta<R_{\text {out }},\end{array}$ 
and

$s_{\max }=\sqrt{R_{\text {out }}^{2}-\zeta^{2}}$

where $R_{\text {out }}$ is the outer edge of the ionized wind. The total observable flux at earth of the wind continuum emission follows from the integration of the wind specific radiation intensity (4) over the wind zone projected to the sky

$F_{\nu, \text { wind }}=\frac{2 \pi}{d^{2}} \int_{0}^{\zeta_{\max }} B_{v}(T)\left(1-\mathrm{e}^{-\tau(\zeta)}\right) \zeta \mathrm{d} \zeta$

where $\zeta_{\max }=R_{\text {out }}$, and $d$ is the distance to the object.

With the wind absorption along each line of sight, i.e., each impact parameter $\zeta$, the stellar flux passing through the wind zone can be calculated from,

$F_{v, \mathrm{star}}=\frac{\pi R_{*}^{2}}{d^{2}} I_{v} \mathrm{e}^{-\tau_{v}(\zeta=0)}$,

with the stellar intensity $I_{v}=4 H_{v}$. For simplicity, we restrict the calculation of the wind attenuation to the direction $\zeta=0$, only. This delivers a lower limit to the real attenuation so that we slightly overestimate the stellar flux leaving the wind zone.

Finally, the total continuum emission is the sum of attenuated stellar and wind contribution, i.e.

$F_{v}=F_{v, \text { star }}+F_{v, \text { wind }}$.

\subsubsection{Gaunt factors for free-free and free-bound processes}

The absorption coefficient of the free-free and free-bound processes, $\kappa_{v}(\zeta, s)\left(\right.$ in $\left.\mathrm{cm}^{-1}\right)$, is given by (see e.g. Brussaard \& van de Hulst 1962)

$\kappa_{v}(\zeta, s) \simeq 3.692 \times 10^{8} \frac{n_{\mathrm{e}}(\zeta, s)^{2}}{v^{3} T^{1 / 2}}\left(1-\mathrm{e}^{-\frac{h \nu}{k T}}\right)\left(g_{\mathrm{ff}}(v, T)+f(\nu, T)\right)$

where $g_{\mathrm{ff}}(v, T)$ is the Gaunt factor for free-free emission and the function $f(v, T)$ contains the Gaunt factors for free-bound processes. To calculate the absorption coefficient, we need to specify the Gaunt factors for both the free-free and the free-bound processes.

In the literature there exist several approximations for the calculation of the free-free Gaunt factors in either the short or the long wavelength regime. To allow for an appropriate transition from one regime to the other (see e.g. Waters \& Lamers 1984; Kraus 2000) we use in the long-wavelength region the relation of Allen (1973), and in the short-wavelength region the expression of Gronenschild \& Mewe (1978). The resulting Gaunt factors are in good agreement with those calculated from the approximation given by Mihalas (1967), which is based on calculations of Berger (1956).

In the top panel of Fig. 2, we plotted the free-free Gaunt factors calculated over a large range of wavelengths and for different electron temperatures. The curves converge to unity for wavelengths shorter than $\sim 1 \mu \mathrm{m}$, while they increase with wavelength in the IR and radio range, where free-free processes are known to dominate the continuum emission. The free-free Gaunt factors thereby depend only weakly on the chosen electron temperature.

The situation is completely different for the function $f(v, T)$, which itself is not a Gaunt factor but that contains the Gaunt factors of the free-bound processes. We calculate this function with

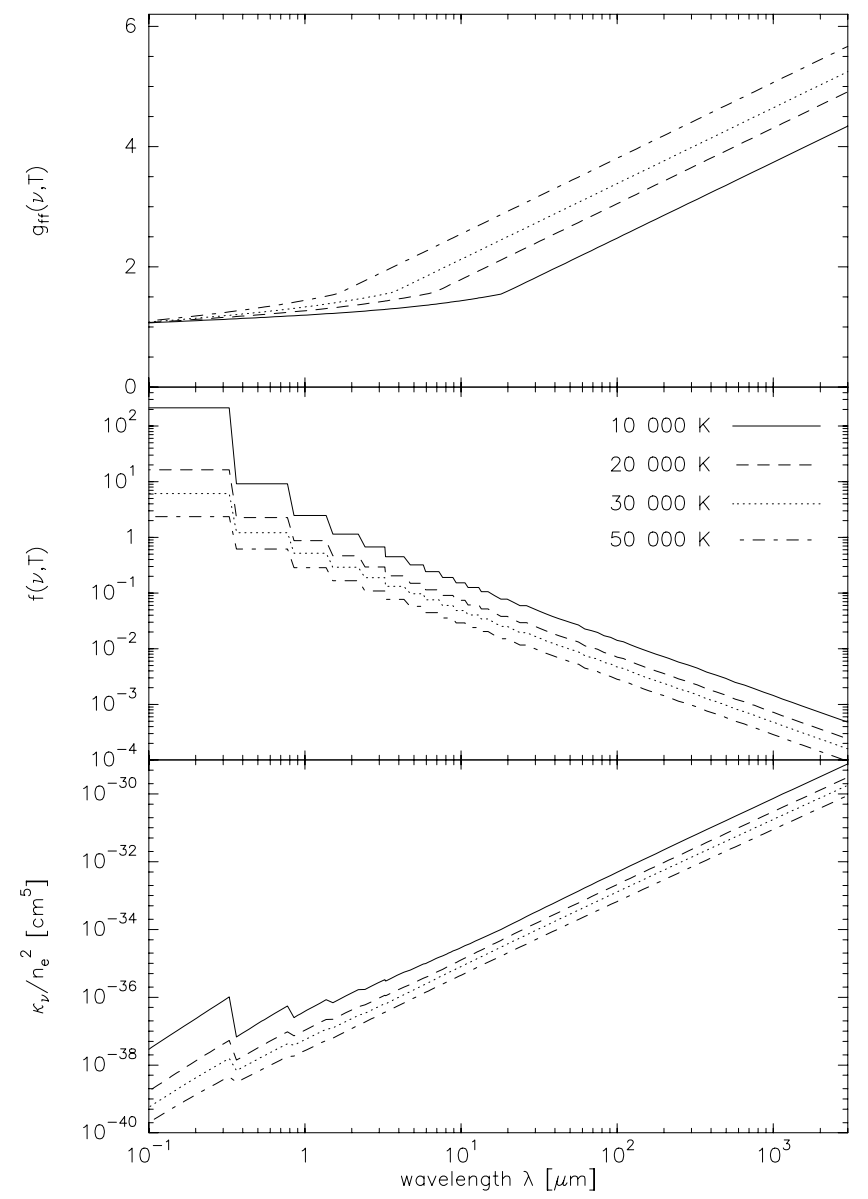

Fig. 2. Top: Gaunt factors for free-free processes. They converge to unity in the optical, but increase with wavelength in the IR and radio range. Middle: the function $f(v, T)$ containing the Gaunt factors for the free-bound processes. The values of $f(v, T)$ increase steeply in the optical, while for $\lambda>1 \mu \mathrm{m}$ they drop quickly and become unimportant compared to the free-free Gaunt factors. Bottom: absorption coefficient of the free-free and free-bound processes (see Eq. (12)). Clearly visible is the growing influence of the free-bound processes in the optical range, especially for decreasing temperature. The line styles indicate the electron temperature.

Eq. (25) of Brussaard \& van de Hulst (1962). The factors $g_{n}$ entering this equation are thereby the Gaunt factors for the transitions from the free level $E=h v_{0} / n^{2}+h v$ to the bound level $E=h v_{0} / n^{2}$. These individual Gaunt factors can, in principle, be calculated with the exact formula given by Menzel \& Pekeris (1935) or by the approximation provided by Mihalas (1967). But it turns out, that these Gaunt factors have values between 0.8 and 1.1 in the wavelength range of our interest (i.e. for $\lambda \gtrsim 0.1 \mu \mathrm{m}$ ) and converge to unity for $\lambda \gtrsim 10 \mu \mathrm{m}$ (see Fig. 8 of Brussaard $\&$ van de Hulst 1962). It is therefore reasonable to use $g_{n} \simeq 1$ for all levels of interest. With this approximation, we calculated the function $f(v, T)$ over the same wavelength range and for the same electron temperatures as the free-free Gaunt factors. The results are shown in the middle panel of Fig. 2.

For $\lambda \gtrsim 1 \mu \mathrm{m}$, the values of $f(v, T)$ are $\ll 1$. The hydrogen free-bound processes, therefore, play no role in the IR and radio regimes. At optical wavelengths however, i.e., for $\lambda<1 \mu \mathrm{m}$, $f(v, T)$ starts to grow steeply, with $f(v, T) \gg 1$ especially for electron temperatures below $\sim 30000 \mathrm{~K}$, i.e., for values typically found in the winds of $\mathrm{OB}$ supergiants. This effect has been mentioned already by Brussaard \& van de Hulst (1962) and 


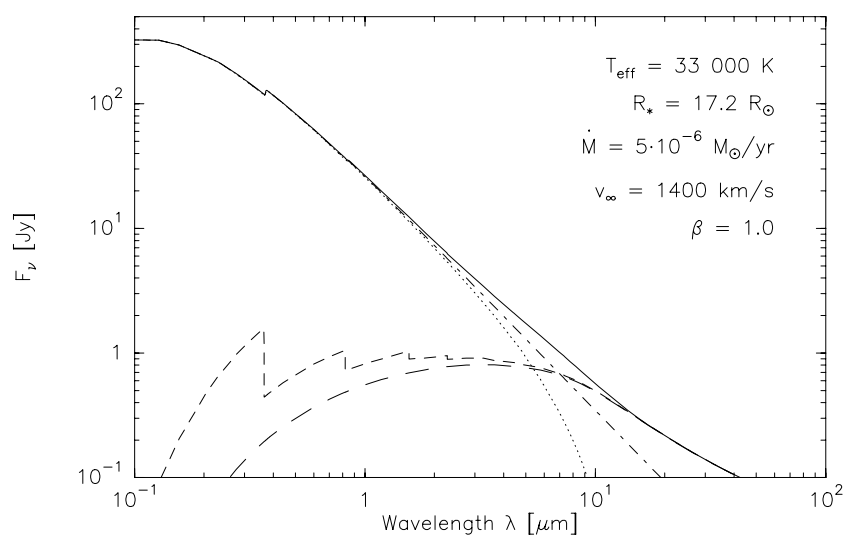

Fig. 3. Continuum emission of a typical OB supergiant (solid line), consisting of the stellar atmosphere having passed through the absorbing wind (dotted) and the thermal wind emission (dashed). Also included is the emission from a wind with pure free-free processes (long-dashed line) and the emission of the star without wind (dashed-dotted).

Kraus (2000) who pointed to the possible importance of the contribution of free-bound processes to the total continuum emission.

The resulting absorption coefficient, $\kappa_{v}$, is shown in the bottom panel of Fig. 2 where we plot $\kappa_{v} / n_{\mathrm{e}}^{2}$ calculated with Eq. (12) for the different electron temperatures. Besides the well-known increase with increasing wavelength, the absorption coefficient also peaks at short wavelengths due to the growing influence of the free-bound processes.

\section{Results}

We fix the terminal wind velocity at $v_{\infty}=1400 \mathrm{~km} \mathrm{~s}^{-1}$ and the mass loss rate at $\dot{M}=5 \times 10^{-6} M_{\odot} \mathrm{yr}^{-1}$. In addition, we place the object to an arbitrary distance of $1 \mathrm{kpc}$. The resulting continuum emission is first calculated for the case of $\beta=1.0$ (Sect. 3.1) before we turn to the more interesting case of higher beta values in Sect. 3.2.

\section{1. $A$ wind with $\beta=1.0$}

For many hot star winds, $\beta$ lies typically in the range $0.8 \ldots 1.0$ (e.g. Markova et al. 2004; Repolust et al. 2004), and we use $\beta=1.0$ for our first test calculation, which serves as a reference model. The resulting spectrum is shown in Fig. 3 where we plot the total continuum emission of our test OB supergiant, consisting of the stellar atmosphere having passed through the absorbing wind and the thermal wind emission. For comparison, we also included the emission of the star without wind. It is obvious, that the wind influences the total spectrum only for $\lambda \gtrsim 1 \mu \mathrm{m}$, while the optical spectrum remains uninfluenced. The stellar continuum only suffers in the IR and radio range, where for $\lambda \gtrsim 10 \mu \mathrm{m}$ the stellar emission is completely absorbed by the wind. In Fig. 3, we also included the results for a wind with pure free-free emission. From a comparison of the total wind emission with the pure free-free wind emission, it is obvious that free-bound processes dominate the wind emission in the optical and near-IR wavelengths, i.e., for $\lambda \lesssim 2 \mu \mathrm{m}$.

To understand where the optical wind continuum is generated, we re-calculate the wind emission for different values of the outer edge of the wind zone. We then compared the resulting emission to the emission from an infinitely large wind, and we plotted the ratios versus wavelength in Fig. 4. From this plot, it

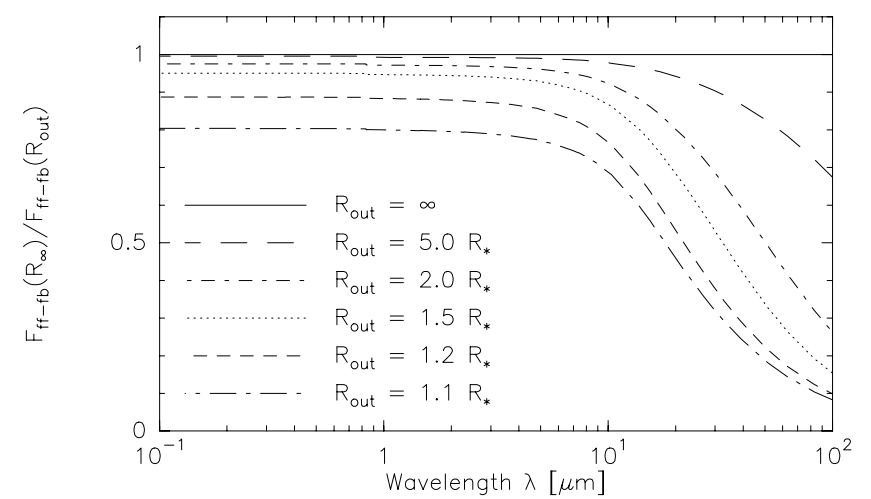

Fig. 4. Increase of the fluxes generated within wind zones with outer edge $R_{\text {out }}$, with respect to an infinitely large wind zone. The thermal wind emission in the optical and near-IR is generated completely in the vicinity of the stellar surface, i.e., within $5 R_{*}$ only.

Table 1. Literature $\beta$ values for OB supergiants. References are: M04 = Markova et al. (2004); C06 = Crowther et al. (2006); K99 = Kudritzki et al. (1999); E04 = Evans et al. (2004); T05 = Trundle \& Lennon (2005); T04 = Trundle et al. (2004).

\begin{tabular}{lccc}
\hline \hline Galaxy & Sp. types & $\beta$ & Ref. \\
\hline Milky Way & O4-O9.7 & $0.7-1.25$ & M04 \\
Milky Way & O9.5-B3 & $1.2-3.0$ & C06 \\
Milky Way & B0-B3 & $1.0-3.0$ & K99 \\
Magellanic Clouds & O8.5-B0.5 & $1.0-3.5$ & E04 \\
Magellanic Clouds & B0.5-B2.5 & $1.0-3.0$ & T05 \\
Magellanic Clouds & B0.5-B5 & $1.0-3.0$ & T04 \\
\hline
\end{tabular}

is evident that the wind emission in the optical and near-IR is generated in the vicinity of the stellar surface, i.e., within $5 R_{*}$. Most of the emission for $\lambda \lesssim 10 \mu \mathrm{m}$ (about 95\%) is even generated within the innermost $1.5 R_{*}$.

\subsection{Winds with $\beta>1.0$}

In recent years, detailed investigations of the wind parameters of OB supergiants have revealed that for many objects the $\beta$ parameter in the velocity law given by Eq. (2) varies over a much larger range. Even values as high as 3.5 are reported. A list of $\beta$ values of $\mathrm{OB}$ supergiants found in the literature is provided in Table 1.

The main effect of a higher $\beta$ value is the less steep increase in the wind velocity with distance from the stellar surface. This is shown in Fig. 5 where we plotted the velocity increase within $5 R_{*}$ from the stellar surface in terms of the terminal velocity for different values of $\beta$. According to the equation of mass continuity (Eq. (1)), the wind density is proportional to $v(r)^{-1}$. Winds with higher $\beta$ values consequently have a (much) higher density in the accelerating wind regions.

We calculated the density distributions in winds with increasing $\beta$ and plotted the densities in terms of the density distribution for the wind with $\beta=1.0 \mathrm{in} \mathrm{Fig.} \mathrm{6.} \mathrm{Close} \mathrm{to} \mathrm{the} \mathrm{stellar} \mathrm{sur-}$ face the densities in the winds with $\beta>1$ are (much) higher than the reference value provided by the wind with $\beta=1$. The higher the $\beta$, the stronger become these relative density enhancements due to (much) slower wind acceleration (see Fig. 5). These relative density enhancements extend over the innermost $\sim 2 \ldots 3 R_{*}$ of the wind, while for larger distances, where the velocity distributions for the higher $\beta$ values also start to approach the terminal velocity, the densities converge. 


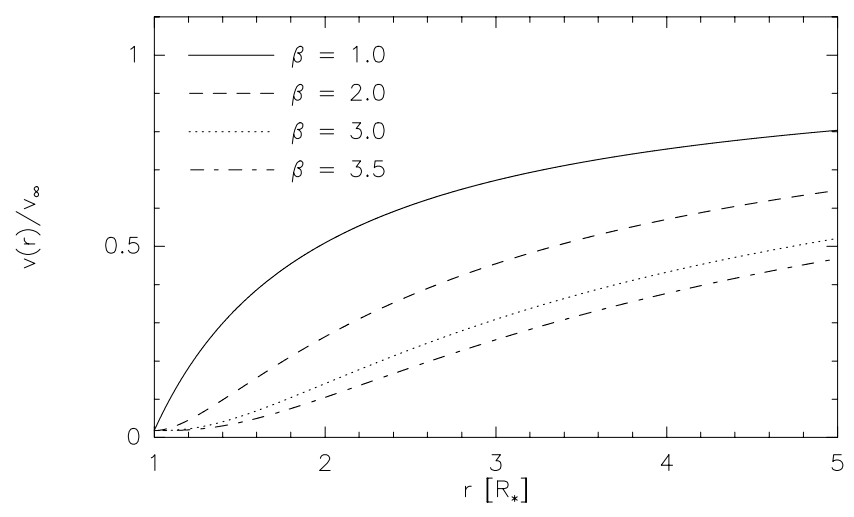

Fig. 5. Velocity increase in winds with different $\beta$ values. The higher the $\beta$, the more slowly the wind is accelerated and the farther away from the star it reaches its terminal value.

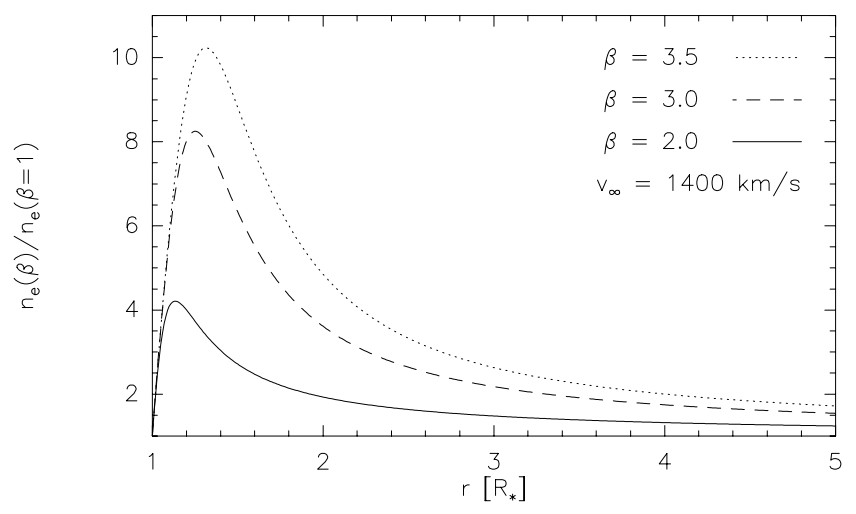

Fig. 6. Density ratios close to the stellar surface for winds with $\beta>$ 1.0 with respect to the wind with $\beta=1.0$. Due to the slower wind acceleration for higher $\beta$, the wind density remains much higher over a larger wind volume, resulting in a relative density enhancement close to the stellar surface, i.e., within $2 \ldots 3 R_{*}$.

In Sect. 3.1 we showed that for a wind with $\beta=1.0$ the optical continuum is generated within $<2 R_{*}$. This is exactly the range where $\beta$ has its strongest influence on the wind density. If the optical continuum for winds with $\beta>1.0$ is generated within approximately the same region, we can expect that the resulting free-bound emission will be strongly influenced by the value of $\beta$.

We determined the wind volume in which the optical flux is generated by comparing the flux generated within an infinitely large wind zone with the flux generated within winds with different outer edges, $R_{\text {out }}$. The results for winds with different values of $\beta$ are shown as a function of the outer edge, $R_{\text {out }}$, in Fig. 7. These results are evaluated for $\lambda=1 \mu \mathrm{m}$ and are also valid for all smaller wavelengths (see Fig. 4). Obviously, for winds with $\beta>1.0$, more than $90 \%$ of the optical continuum is generated within $1.5 R_{*}$, and more than $98 \%$ are produced within $2 R_{*}$.

Before we turn to the calculation of the total continuum emission from winds with $\beta>1.0$, we first investigate the influence of $\beta$ on the stellar emission. Since the wind absorption coefficient, $\kappa_{\mathrm{ff}-\mathrm{fb}}$, is proportional to $n_{\mathrm{e}}^{2}$ (see Eq. (12)), an increasing density leads to a (strongly) increasing optical depth. The stellar flux will therefore be more strongly absorbed in the wind with a high $\beta$ compared to a wind with $\beta=1$. This is illustrated in Fig. 8. Of course, the strongest depression of the stellar emission occurs at IR wavelengths $(\lambda>1 \mu \mathrm{m})$; but with increasing $\beta$ some part of the stellar flux at optical wavelengths, especially in the red part of the spectrum, is also absorbed.

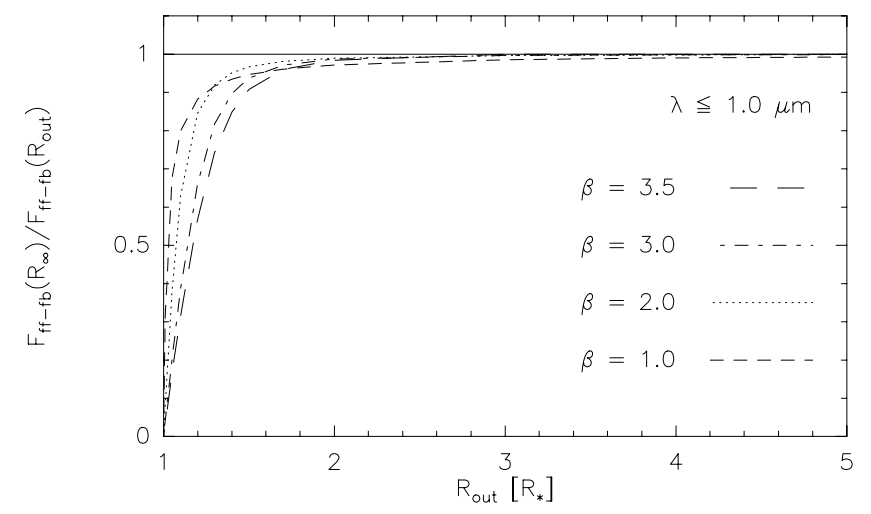

Fig. 7. Flux ratio $F_{\mathrm{ff}-\mathrm{fb}}\left(R_{\infty}\right) / F_{\mathrm{ff}-\mathrm{fb}}\left(R_{\mathrm{out}}\right)$ as a function of outer wind edge $R_{\text {out }}$ for different values of $\beta$. The calculations shown are for $\lambda=1 \mu \mathrm{m}$ and are also valid for all smaller wavelengths. As reference, we include the ratio for $R_{\text {out }}=R_{\infty}$ (solid line). For all values of $\beta$, the optical wind continuum emission is generated well within the plotted wind size of $5 R_{*}$.

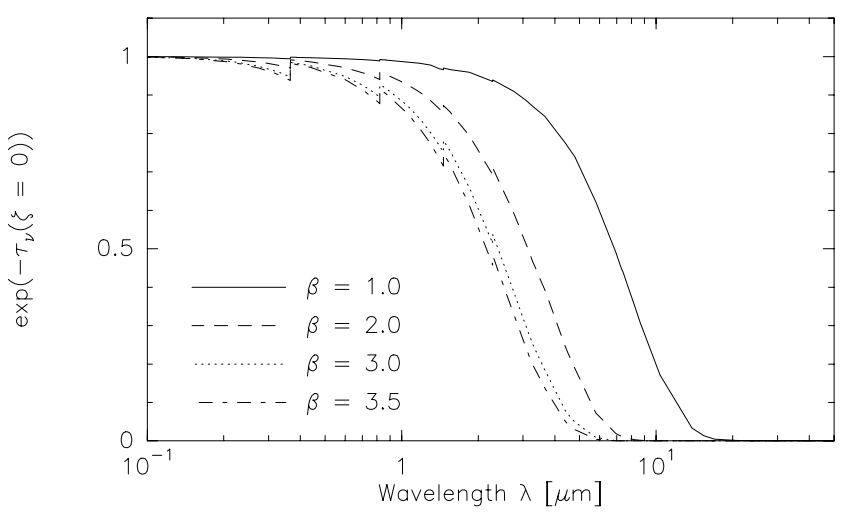

Fig. 8. Fraction of stellar radiation escaping from the wind zone for different values of $\beta$. While for $\beta=1.0$ all the wind continuum emission for $\lambda<1 \mu \mathrm{m}$ passes unabsorbed through the wind, the situation is different for higher $\beta$ values for which even the optical wind continuum suffers from wind absorption along its way through the wind. The higher $\beta$ the more stellar emission is absorbed also from the near- and mid-IR stellar spectrum.

But while the increasing absorption coefficient results in a decrease of the stellar flux, it leads at the same time to a strong increase of the free-free and free-bound emission in the optical and IR part of the spectrum. This can be seen upon inspection of Fig. 9 where we plotted the total continuum emission for winds with different $\beta$ values. The optical wind continuum increases with $\beta$ leading to an enhanced total continuum in the IR and even at optical wavelengths. This increase, especially at optical wavelengths, is more evident in Fig. 10. There we plot the flux ratio between the total continuum emission of the star plus wind system with $\beta>1$ and the total continuum emission of the system with $\beta=1$ as a function of wavelength. For $\beta \gtrsim 3.0$, the total continuum exceeds the one for $\beta=1$ in the optical by about $15 \%$ in the blue spectral range and by about $25-30 \%$ in the red spectral range, while in the near- and mid-IR it reaches even more than $200 \%$.

The contribution of the pure wind emission to the total continuum production is finally shown in Fig. 11. Its importance grows and shifts to lower wavelengths with increasing $\beta$. While for $\beta=1.0$ the contribution to the optical is less than $1 \%$, it grows to $>10 \%$ for $\beta=2.0$ and to values exceeding even $30 \%$ for $\beta \gtrsim 3.0$. 


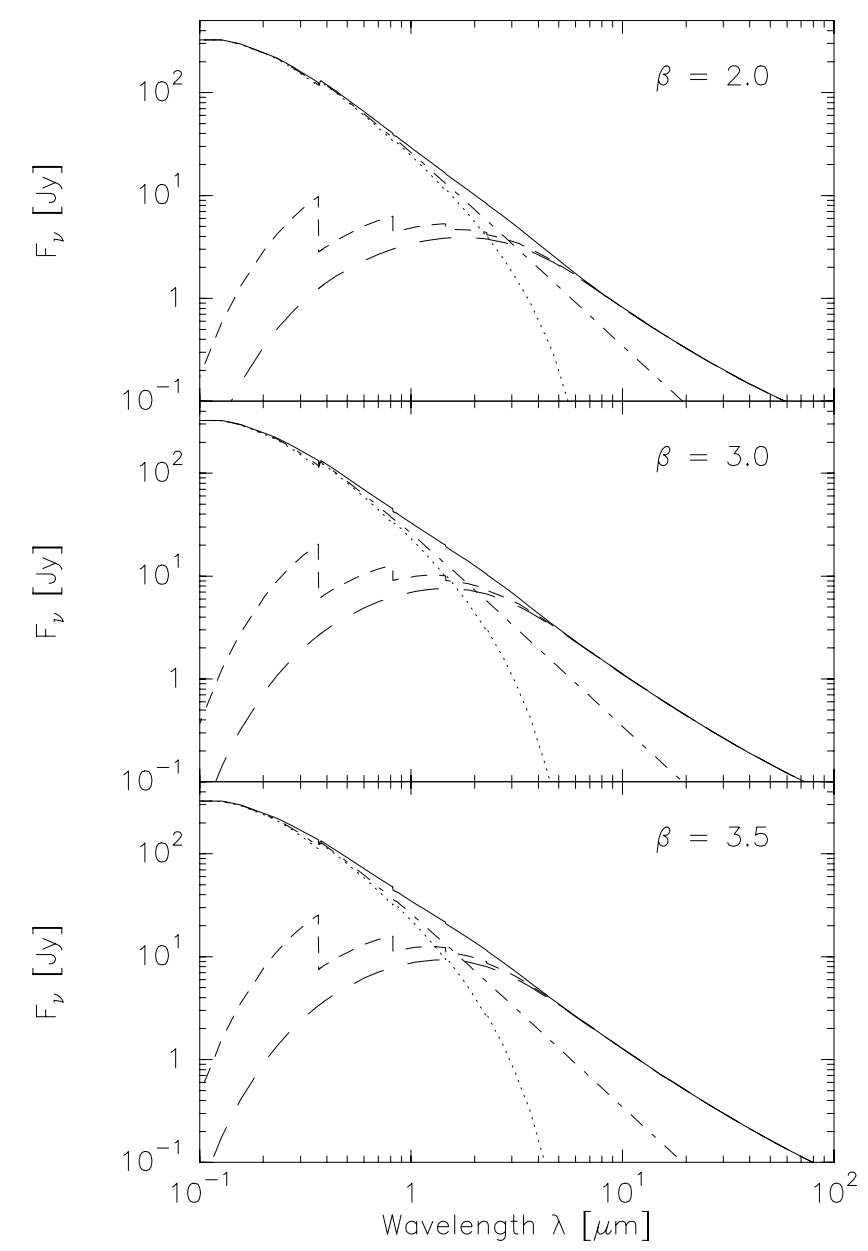

Fig. 9. Comparison of total continuum spectra of the star plus wind system (solid lines) for different values of $\beta$. As in Fig. 3, a wind with pure free-free emission is included in each panel (long-dashed line) to emphasize the growing importance of the free-bound contributions for $\lambda \leq 2 \mu \mathrm{m}$. With increasing $\beta$ the free-free and free-bound contributions (dashed) increase especially in the near-IR and optical spectrum. At the same time, the stellar continuum (dotted) suffers from the increasing absorptivity of the wind zone. For comparison, the pure stellar continuum is included (dashed-dotted).

In summary, we can therefore state that even at optical wavelengths the wind plays a non-negligible role for the continuum emission of $\mathrm{OB}$ supergiants having wind velocity distributions with $\beta>1.0$.

\section{Discussion}

For our calculations we made use of several assumptions and simplifications like a constant wind temperature, the core-halo approximation, LTE even for the bound-free processes, and the neglect of electron scattering. These are severe restrictions to keep the model as simple as possible. Here, we want to discuss the influence and possible consequences of these assumptions and simplifications on the model results. In addition, we want to address shortly the topic of wind clumping and its expected influence on our results.

\subsection{The wind temperature}

For our model calculations of stellar wind emission (see Sect. 2.2) we made two severe assumptions concerning the

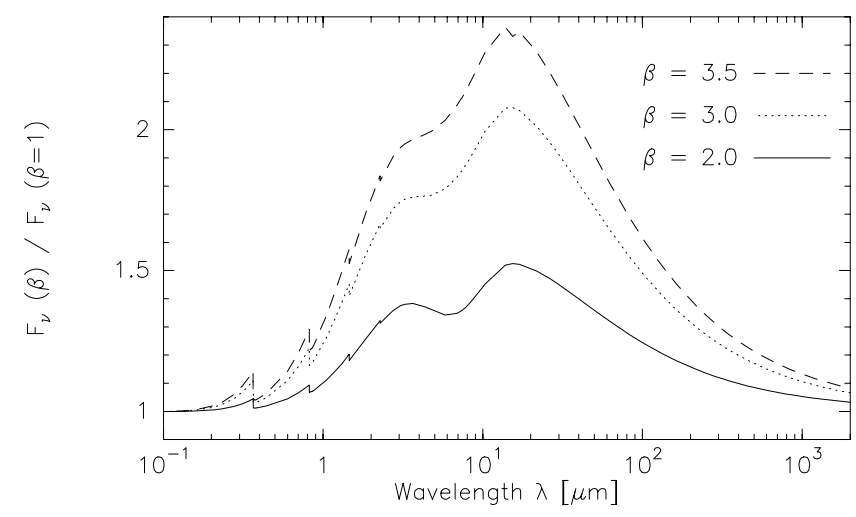

Fig. 10. Continuum flux ratio of the star plus wind system for different values of $\beta$ with respect to $\beta=1$.0. With increasing $\beta$ the increase in optical flux due to the increasing importance of the free-bound emission from the wind becomes visible.

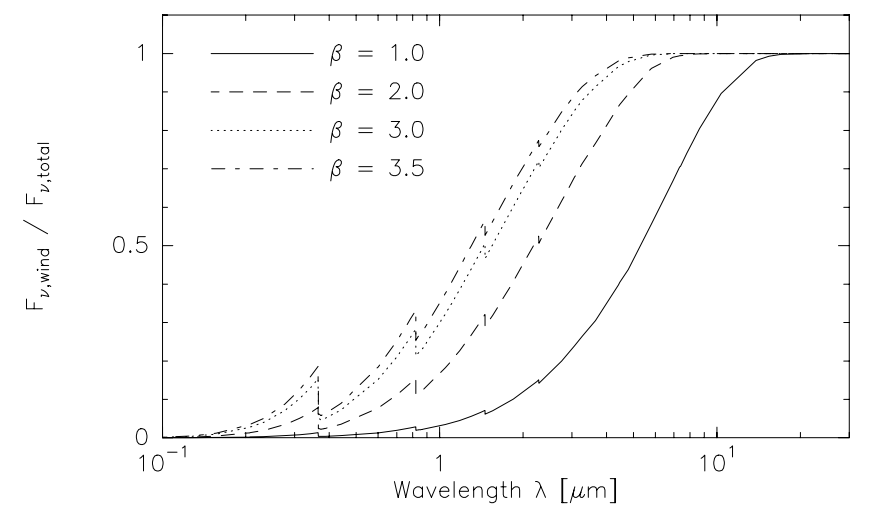

Fig. 11. Ratio of the wind emission with respect to the total continuum emission for different values of $\beta$. While for $\beta=1.0$ the wind contribution to the total optical continuum is less than $1 \%$, its influence grows quickly with increasing $\beta$, reaching values up to $30 \%$ in the red part of the optical spectrum for $\beta>3.0$.

temperature: (i) we assumed the wind to be isothermal; and (ii) we used a rather high temperature, i.e. the temperature at $r=R_{*}$, as the global electron temperature in the wind. Here, we want to discuss why these two assumptions are reasonable.

Our choice of the electron temperature at $r=R_{*}$ means that we are calculating a lower limit of the wind absorption coefficient, $\kappa_{v}$, because it is proportional to $T^{-1 / 2}$ and the Gaunt factors increase with decreasing temperature (see Fig. 2). Consequently, according to Eqs. (4) and (9), the emission caused by freefree and free-bound processes, equally increases with decreasing electron temperature. Our results calculated for the high electron temperature thus underestimate the real wind continuum emission. The error in wind emission can be estimated by looking at the real expected temperature distribution within the region where the optical wind continuum is generated.

During our investigations we found that for each value of $\beta$, $95 \%$ of the wind continuum at optical wavelengths is generated in the vicinity of the stellar surface, i.e. within $1.40 \ldots 1.65 R_{*}$. This is shown in the top panel of Fig. 12. The solid horizontal line in this plot indicates the $95 \%$ level of the emission, and the vertical lines indicate, for each $\beta$, the distance at which this level is reached.

To see how,in a more realistic wind model, the temperature would have changed over the wind region in which the optical continuum emission is generated, we finally calculate the 


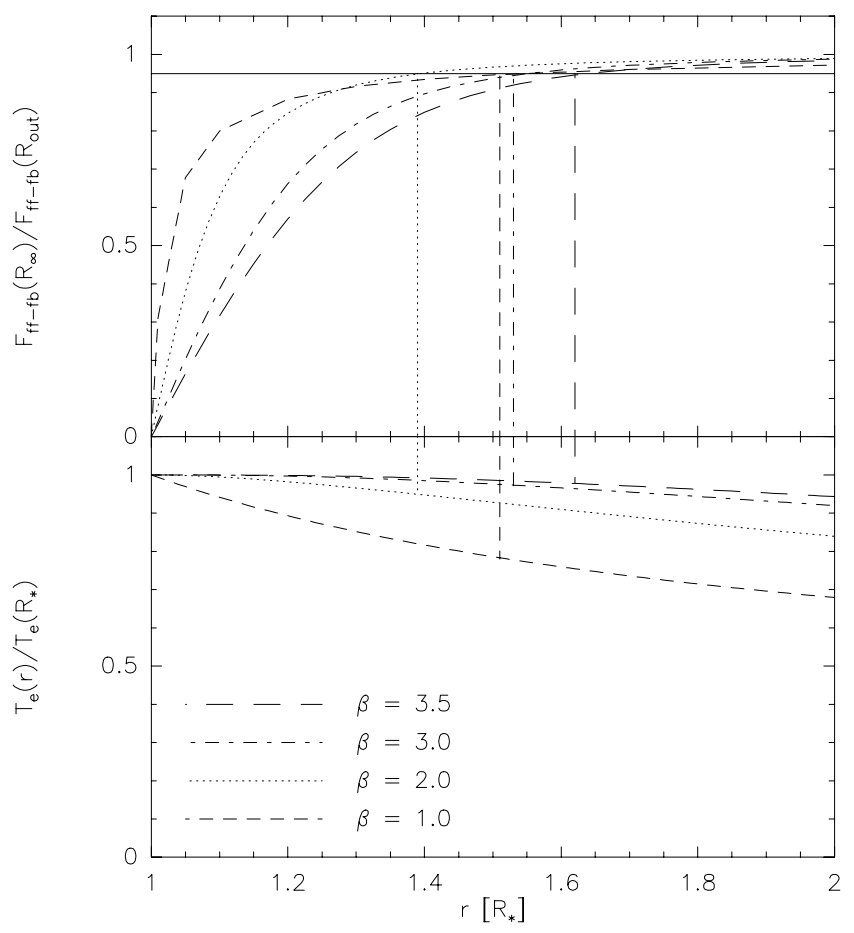

Fig. 12. Top: as Fig. 7 but for the innermost $2 R_{*}$, only. The solid horizontal line indicates the $95 \%$ level, and the vertical lines the distances where, for winds of different $\beta$ values, this level is reached. Bottom: electron temperature distribution within $2 R_{*}$ for different values of $\beta$, normalized to the maximum electron temperature. For $\beta>1.0$, the inner parts of the winds remain much hotter and almost at a constant value compared to the wind with $\beta=1.0$.

temperature distribution according to Eq. (3), with the velocity distribution as defined by Eq. (2). The resulting temperature distributions for different values of $\beta$ are shown in the bottom panel of Fig. 12. An increase in $\beta$ results in a lower wind velocity at the same distance from the star and, after Eq. (3), in a higher wind temperature. Therefore, the higher the $\beta$, the hotter the wind remains at the same location.

For winds with $\beta \geq 2.0$, the drop in wind temperature is found to be less than $5 \%$ within the free-free and free-bound emission generation zone. Such a small decrease in temperature results in only a tiny and therefore negligible increase in wind emission. The assumption of an isothermal wind with $T_{\mathrm{e}}=T_{\mathrm{e}}\left(R_{*}\right)$ is therefore well justified.

The situation is different for winds with $\beta=1.0$. Here, the drop in wind temperature is found to be on the order of $20 \%$, which corresponds to a decrease in electron temperature by about $5000 \mathrm{~K}$ for our chosen model star. In such a case, the temperature distribution in the wind is not negligible. The decrease in electron temperature results in an increase of the wind absorption coefficient of about a factor of two (see Fig. 2), and consequently to a noticeably enhanced wind emission. However, even with an enhanced wind emission, the stellar spectrum still clearly dominates the total optical continuum. For OB supergiants with $\beta=1.0$, the assumption of an isothermal wind with $T_{\mathrm{e}}=T_{\mathrm{e}}\left(R_{*}\right)$ is therefore still an acceptable approximation, as long as the mass loss rate of the star is not extremely high, as in the case of Luminous Blue Variables or B[e] supergiants.

\subsection{Core-halo approximation}

The construction of our star plus wind model is often referred to as the core-halo approximation, i.e., it is assumed that the stellar atmosphere is in hydrostatic equilibrium, and the wind is treated seperately with a density distribution following from the mass continuity equation. With such a treatment the transition between the atmosphere and the wind is not properly accounted for.

However, the intention of our research was to investigate the influence of one single parameter, namely the steepness of the velocity increase, $\beta$, keeping the stellar and the remaining wind parameters fixed. Deviations in density distribution of the star plus wind system introduced by the use of the core-halo approximation will consequently appear in all our discussed models and will influence all our results in the same way. But they will not significantly alter our conclusions, which are mainly based on the comparison of the spectra (i.e. the ratios) for stars with different $\beta$ values.

\subsection{Non-LTE effects and electron scattering}

In our calculations, we assumed the wind to be in LTE. This is a justified assumption for the free-free processes since they are collisional. For the bound-free processes the situation is less clear. Here, non-LTE effects might play an important role. According to our non-LTE model atmosphere calculations (e.g. Kubát 2003), departure coefficients can be (much) larger than unity in the outer parts of the stellar atmosphere (especially for the ground level) and, consequently, they lead to a higher boundfree opacity.

We tested the influence of non-LTE effects on our results in a qualitative way by increasing artificially the bound-free opacity. This results in an increase in wind optical depth and, according to Eq. (10), to an increased attenuation of the stellar emission, while the wind emission remains largely unaffected. Consequently, the flux of the total continuum emission decreases with increasing bound-free opacity, i.e., increasing influence of non-LTE effects. At the same time, the importance of the wind emission with respect to the total continuum increases. The neglect of non-LTE effects thus results in a lower limit of the wind contribution.

A similar conclusion can be drawn from the neglect of electron scattering. Since electron scattering attenuates the stellar light passing through the wind zone, it equally acts as an additional opacity source. The consequences for the total continuum emission are therefore the same: a decrease in total emission (over the wavelength ranges that are dominated by the stellar emission), and simultaneously an increase of the wind emission with respect to the total continuum.

For our model star plus wind system, we checked the electron scattering optical depth in radial direction. We find that for the chosen stellar and wind parameters and the different $\beta$ values, the electron scattering optical depth is always smaller than unity. However, compared to the free-bound opacity, it is not negligible. This means, that we calculated indeed upper limits for the stellar emission resulting in lower limits for the wind emission with respect to the total continuum. A proper treatment of nonLTE effects and electron scattering can therefore only confirm our results: the importance of the wind contribution to the total optical continuum emission. 


\subsection{Wind clumping}

In recent years, observational and theoretical investigations provided evidence of wind clumping (see e.g. Hillier 2005). One of the most striking results found from detailed spectroscopic analyses (e.g. Crowther et al. 2002; Hillier et al. 2003; Bouret et al. $2003,2005)$ was that if a wind is clumped, the mass loss rates inferred from spectroscopy might be lower on average by a factor of 3. In addition, Hillier et al. (2003) found that clumping starts close to the photosphere, i.e., within the region in which the influence of $\beta$ is strongest.

The wind emission calculated in our study is not only determined by the $\beta$ parameter of the velocity distribution, but also by the mass loss rate of the star that was kept constant during our analysis. Many of the authors who derived the high $\beta$ values (see Table 1) also claim that their mass loss rates are derived under the assumption of unclumped winds and might well be a factor of 3 lower. Therefore, the question arises how our results might change if we account for wind clumping.

This paper is not aimed to study wind clumping in detail, instead we refer to the recent paper by Kraus et al. (2008) who investigated in more detail the influence of wind clumping on the optical continuum emission of OB supergiants. Their results can be summarized as follows: wind clumping, introduced into the calculations, e.g., by the filling factor approach of Hiller et al. (2003), results in a slight decrease in wind emission at optical wavelengths. This decrease, however, was found to be less than the increase of the wind emission due to a high $\beta$ value compared to a wind with $\beta=1.0$. We can, therefore, conclude that in clumped winds with high $\beta$ values the effects discussed in this paper are still present, but probably slightly weakened.

\section{Conclusions}

We investigated the influence of the thermal wind emission produced by free-free and free-bound processes to the total continuum of normal OB supergiants. While for winds with a velocity distribution following a $\beta$-law with $\beta$ typically in the range of 0.8 to 1.0 , no influence of the wind at optical wavelengths is expected, the situation can be different when $\beta$ exceeds 1.0. High $\beta$ values reaching even 3.5 have recently been found for many OB supergiants. Our investigations therefore concentrated on such high beta values and their influence on the wind continuum emission especially at optical wavelengths.

We found that the wind emission in the optical is generated within $2 R_{*}$, only. This region is exactly the region where $\beta$ has its highest influence on the wind density structure. Since with increasing $\beta$ the wind is accelerated much more slowly than for $\beta=1.0$, the density close to the stellar surface is strongly enhanced, leading to an enhanced production of especially freebound emission at optical wavelengths. At the same time the stellar emission, which passes through the wind on its way to the observer, is absorbed. These effects increase with increasing $\beta$. The total continuum of OB supergiants, for which $\beta$ values higher than 1.0 are found, can thus contain non-negligible contributions of wind emission even at optical wavelengths.

Acknowledgements. We thank the referee, Ian Howarth, for his comments. M.K. acknowledges financial support from GA AV ČR under grant number KJB300030701.

\section{References}

Allen, C. W. 1973, Astrophysical Quantities (London: The Athlone Press) Berger, J. M. 1956, ApJ, 124, 550

Bouret, J.-C., Lanz, T., Hillier, D. J., et al. 2003, ApJ, 595, 1182

Bouret, J.-C., Lanz, T., \& Hillier, D. J. 2005, A\&A, 438, 301

Bresolin, F., Kudritzki, R. P., Lennon, D. J., et al. 2002, ApJ, 580, 213

Brussaard, P. J., \& van de Hulst, H. C. 1962, Rev. Mod. Phys., 34, 507

Bunn, J. C., \& Drew, J. E. 1992, MNRAS, 255, 449

Crowther, P. A., Hillier, D. J., Evans, C. J., et al. 2002, ApJ, 579, 774

Crowther, P. A., Lennon, D. J., \& Walborn, N. R. 2006, A\&A, 446, 279

de Koter, A. 1993, Ph. D. Thesis, Utrecht University, The Netherlands

Drew, J. E. 1989, ApJS, 71, 267

Evans, C. J., Crowther, P. A., Fullerton, A. W., \& Hillier, D. J. 2004, ApJ, 610, 1021

Fullerton, A. W., Massa, D. L., \& Prinja, R. K. 2006, ApJ, 637, 1025

Gronenschild, E. H. B. M., \& Mewe, R. 1978, A\&AS, 32, 283

Guo, J. H., \& Li, Y. 2007, ApJ, 659, 1563

Hillier, D. J. 2005, in The fate of the most massive stars, ed. R. Humphreys, \& K. Stanek (San Francisco: ASP), ASP Conf. Ser., 332, 219

Hillier, D. J., Lanz, T., Heap, S. R., et al. 2003, ApJ, 588, 1039

Kraus, M. 2000, Ph.D. Thesis, University of Bonn, Germany

Kraus, M., Borges Fernandes, M., \& de Araújo, F. X. 2007, A\&A, 463, 627

Kraus, M., Kubát, J., \& Krtička, J. 2008, in Clumping in Hot Star Winds, ed. W.-R. Hamann, A. Feldmeier, \& L. Oskinova (Potsdam: Univ.-Verl.), in press [arXiv:0708.0735]

Krtička, J. 2006, MNRAS, 367, 1282

Kubát, J. 2003, in Modelling of Stellar Atmospheres, ed. N. Piskunov, W. W. Weiss, \& D. F. Gray (San Francisco: ASP), IAU Symp., 210, A8

Kudritzki, R. P., Puls, J., Lennon, D. J., et al. 1999, A\&A, 350, 970

Lamers, H. J. G. L. M., \& Cassinelli, J. P. 1999, Introduction to stellar winds (Cambridge University Press)

Lamers, H. J. G. L. M., \& Leitherer, C. 1993, ApJ, 412, 771

Markova, N., Puls, J., Repolust, T., \& Markov, H. 2004, A\&A, 413, 693

Menzel, D. H., \& Pekeris, C. L. 1935, MNRAS, 96, 77

Mihalas, D. 1967, ApJ, 149, 169

Olnon, F. M. 1975, A\&A, 39, 217

Panagia, N., \& Felli, M. 1975, A\&A, 39, 1

Puls, J., Kudritzki, R.-P., Herrero, A., et al. 1996, A\&A, 305, 171

Puls, J., Markova, N., Scuderi, S., et al. 2006, A\&A, 454, 625

Repolust, T., Puls, J., \& Herrero, A. 2004, A\&A, 415, 349

Trundle, C., \& Lennon, D. J. 2005, A\&A, 434, 677

Trundle, C., Lennon, D. J., Puls, J., \& Dufton, P. L. 2004, A\&A, 417, 217

Waters, L. B. F. M., \& Lamers, H. J. G. L. M. 1984, A\&AS, 57, 327 